\title{
Simultaneous alignment and Lorentz angle calibration in the CMS silicon tracker using Millepede II
}

\author{
Nazar BARTOSIK*† \\ Deutsches Elektronen-Synchrotron \\ E-mail: nazar.bartosikecern.ch
}

The CMS silicon tracker consists of 25684 sensors that provide measurements of trajectories of charged particles that are used by almost every physics analysis performed by CMS. In order to achieve high measurement precision, the positions and orientations of all sensors have to be determined very accurately. This is achieved by track-based alignment using the global fit approach of the Millepede II program. This approach is capable of determining about 200000 parameters simultaneously. The alignment precision has now reached such a high level that even small calibration inaccuracies are noticeable. As such, the alignment framework has been extended to treat position sensitive calibration parameters. Of special interest is the Lorentz angle which affects the hit positions due to the drift of the signal electrons in the magnetic field. We present a general description of the alignment and calibration procedure, as well as results from measurements of the Lorentz angle and its time dependence during the full 2012 data taking period.

The European Physical Society Conference on High Energy Physics

18-24 July, 2013

Stockholm, Sweden

\footnotetext{
*Speaker.

†n behalf of the CMS Collaboration.
} 


\section{Introduction}

Almost all physics analyses performed by the Compact Muon Solenoid (CMS) experiment rely heavily on measurements made by the tracking detector, requiring the highest level of precision. To reach the required resolution of track transverse momentum $\left(p_{\mathrm{T}}\right)$ the tracker is enclosed in a superconducting solenoid that produces a strong magnetic field (3.8T) to bend trajectories of charged particles. The detector is equipped with 25684 silicon sensors that can be divided into two main groups: pixel sensors (2D measurements) and microstrip sensors (1D measurements).

Pixel sensors in the central region are organized in the pixel barrel (BPIX) subdetector, constructed of 3 concentric layers. Each layer consists of 8 rings of sensors along the beampipe [1]. Track reconstruction precision depends heavily on the accuracy of the alignment of the tracker, as it determines coordinates of the centre of each sensor, its rotational angles and surface deformations.

\section{Track-based alignment with Millepede II algorithm}

The geometry of the detector is determined from a large sample of reconstructed trajectories of charged particles. This alignment technique is based on the fact that the distribution of residuals between the measured and predicted hit positions becomes generally broader if the assumed geometry differs from the real one. The task of the alignment algorithm is to minimise the sum of the squares of the normalised residuals, measured using a large number of tracks:

$$
\chi^{2}(\mathbf{p}, \mathbf{q})=\sum_{j}^{\text {tracks }} \sum_{i}^{\text {measurements }}\left(\frac{m_{i j}-f_{i j}\left(\mathbf{p}, \mathbf{q}_{j}\right)}{\sigma_{i j}}\right)^{2}
$$

In Equation $2.1 f_{i j}$ represents the track model prediction of the measured hit position, which depends on the geometry parameters $(\mathbf{p})$ and track parameters $\left(\mathbf{q}_{j}\right)$. Linearising $f_{i j}$ around initial values transforms the problem into a system of matrix equations that can be solved numerically.

With 25684 sensors in the CMS tracker and up to 9 alignment parameters per sensor, the total number of fit parameters can exceed 200 000, requiring a large sample of tracks. To solve this problem, the Millepede II program is used, employing the global fit approach. This method can still be insensitive to particular systematic distortions of the geometry (weak modes) which do not increase the residuals but degrade the physics performance [1]. In order to control these weak modes, samples with different track topologies are used. These include tracks from isolated muons, muons from $Z \rightarrow \mu \mu$ decays, as well as low $p_{\mathrm{T}}$ tracks and cosmic rays.

\section{Lorentz angle calibration}

When a charged particle traverses a silicon sensor, it releases charge carriers that drift under the electric field in the direction normal to the sensor surface. If the sensor is located in the magnetic field, the Lorentz force deflects the signal charge by Lorentz angle $\left(\theta_{L A}\right)$. This leads to an increase in cluster size and a shift of its centre in the local $\mathrm{X}$ direction by $\Delta x$, which depends on the thickness of the sensor and the Lorentz angle. The latter is parameterized by the mobility $(\mu)$ and the magnetic field in the local Y direction $\left(B_{y}\right)$ :

$$
\Delta x=\tan \left(\theta_{L A}\right) \frac{d}{2}, \quad \text { where } \theta_{L A}=\mu B_{y}
$$


The CMS alignment software, which is based on Millepede II, can in principle determine any parameter that measured hit positions depend on. Therefore the Lorentz angle calibration has been integrated to the alignment procedure. Since the Lorentz angle effect is present only in a magnetic field, cosmic and low $p_{\mathrm{T}}$ tracks recorded without magnetic field have been added in order to disentangle it from alignment.

The Lorentz angle has been calibrated for 24 groups of pixel sensors: 8 rings $\times 3$ layers. The 2012 data-taking period has been divided into 65 time intervals of similar integrated luminosity $\left(\sim 330 \mathrm{pb}^{-1}\right)$ to check for time dependence and hence a dependence on the accumulated irradiation dose. The evolution of the calibrated Lorentz angle is shown in Figure 1. Within a single layer the Lorentz angle develops consistently in each of 8 rings with an offset between negative $(Z<0)$ and positive $(Z>0)$ parts of the barrel of the pixel detector. In each layer, on the other hand, the evolution has a different shape, which can be a manifestation of the same behaviour, delayed in more distant layers.
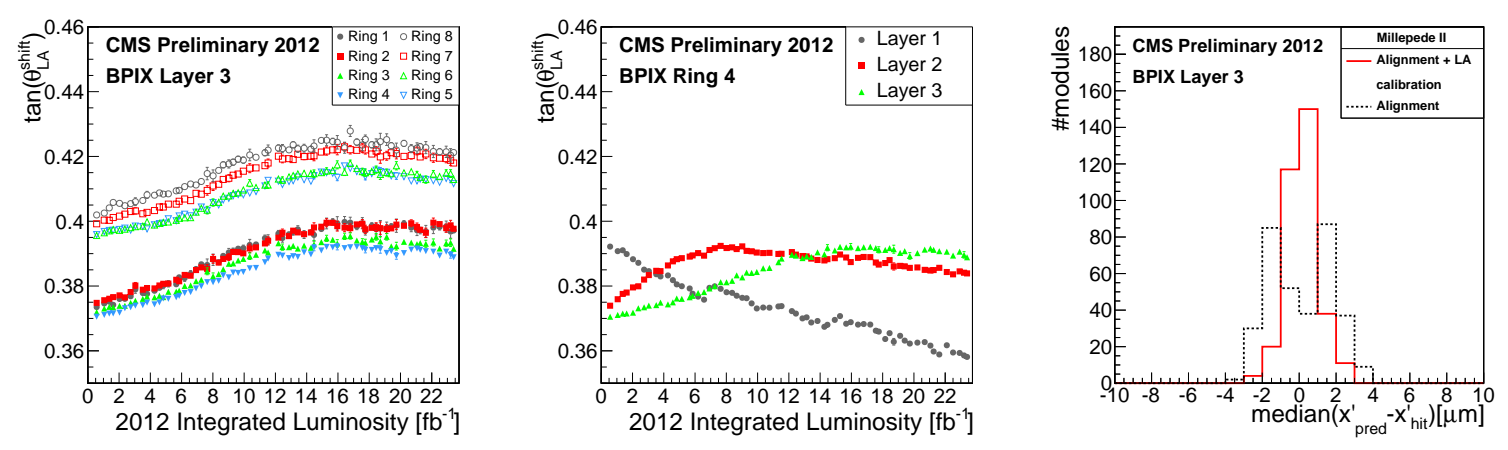

Figure 1: Evolution of the Lorentz angle in each ring of layer 3 (left) and in ring 4 of each layer (middle) of the BPIX. Distribution of the median of the residuals for modules in layer 3 of the BPIX (right).

In order to verify the calibrated Lorentz angle values, track-hit residuals of 2 million tracks from isolated muons have been analysed. For each module the median of the residuals has been calculated. The right-most plot in Figure 1 shows this distribution for the geometry obtained from alignment alone and the one obtained from simultaneous alignment and calibration. The latter has a narrower distribution, which means that the corresponding geometry is closer to the true one.

\section{Summary}

The alignment methodology of the CMS silicon tracker alignment has been extended to simultaneously calibrate the Lorentz angle in the barrel pixel detector, such that local variations and time dependence can be studied in great detail. This approach improves the overall precision of the hit reconstruction and allows consistent use of $0 \mathrm{~T}$ and $3.8 \mathrm{~T}$ data in the alignment procedure. The importance of this improvement will be particularly relevant during the next run of the LHC, when larger irradiation doses are expected to effect stronger variations of the Lorentz angle.

\section{References}

[1] J. Hauk, "Measurement of associated $Z^{0}$-boson and b-jet production in proton-proton collisions with the CMS experiment". PhD thesis, Universität Hamburg, CMS-TS-2012-027 (2012). 